\title{
Tres nuevas especies de Anthurium, (Araceae), para Colombia, Tolima, Ibagué, Cañon del Combeima
}

Three new species of Anthurium, (Araceae), for Colombia, Tolima, Ibagué, Combeima Canyon

\author{
German Orlando Oyuela Torres ${ }^{1}$ \& Thomas B. Croat $^{2,3}$
}

\begin{abstract}
Resumen
Tres nuevas especies son descritas para Colombia, Anthurium combeimense, A. oyuelae y A. sanjorgense, plantas endémicas de la región del cañón del río Combeima en el municipio de Ibagué del departamento del Tolima. Una especies es un miembro de la sección Pachyneurium, series Pachyneurium caracterizado por sus hojas en forma de roseta, internados cortos y raíces compacto. Dos especies son miembros de sect. Calomystrium caracterizado por sus catafilos intactos y hojas ovato-sagitata.

Palabras-claves: Araceae, Colombia, nueva species, departamento de Tolima.
\end{abstract}

\begin{abstract}
Three new species are described for Colombia, Anthurium combeimense, A. oyuelae and A. sanjorgense. The species are endemic to the valley of the Río Combeima in the municipality of Ibagué in Tolima Department. One species is a member of section Pachyneurium, series Pachyneurium, characterized by rosulate leaves, short internodes and dense roots. Two species are members of sect. Calomystrium characterized by their long-petiolate leaves, persistent intact cataphylls and ovate-sagittate leaf blades.
\end{abstract}

Key words: Araceae, Colombia, new species, Tolima Department.

\section{Introducción}

La familia Araceae comprende cerca de 122 géneros y un estimado 5500 especies herbáceas y trepadoras (Boyce \& Croat 2011). Las aráceas se distribuyen en muchos lugares del mundo en especial en zonas con temperaturas altas, para América las Araceae se distribuyen desde el norte de México y las Antillas mayores a sur de Brasil y norte de Argentina y Uruguay, que se producen en hábitats abiertos o forestales. Pueden ser terrestres, epífitas, hemiepífitas, rupícolas o acuaticas (Croat 1988(1990); Mayo et al. 1997). En Colombia hay muchas zonas que no han sido estudiadas y no hay un número exacto de especies de anthurios descritas. En Brasil los estudios arrojan, alrededor de 130 especies de Anthurium, es un país con gran diversidad de este género (Temponi \& Coelho 2011) y es de anotar que hay anthurios que su distribución geográfica puede alcanzar dos países, como ocurre con muchos anthurios que han sido ubicados en Panamá, pero también son encontrados en las selvas del Choco, un ejemplo de ello es el caso de Anthurium obtusum y A. crystallinum Linden \& André que por muchos años se creyó que era originario de Panamá pero se ha concluido que es originario de Colombia.

El género Anthurium ha sido dividido en 18 secciones (Croat \& Sheffer 1983), Tetraspermium Schott, Gymnopodium Engl., Porphyrochitonium Schott, Digitinervium Sodiro, Pachyneurium Schott, (sección Pachyneurium, series Pachyneurum, series, Multinervia), Polyphyllium Engl., Leptanthurium Schott, Decurrentia Schott, Xialophyllium Schott, Polyneurium Engl, Urospadix Engl, Cardiolonchium Schott, Chamaerepium Schott, Calomystrium Schott corregido Engl., Belolonchium Schott corregido Engl., Semaeophyllium Schott, Schizoplacium Schott y Dactylophyllium Schott.

La familia Araceae se encuentra distribuida en todo Colombia debido a las condiciones climáticas

\footnotetext{
' Jardín Botánico San Jorge, Vía Calambeo, Antigua Granja San Jorge, C.P. 730001, Ibagué, Tolima, Colombia.

${ }^{2}$ Missouri Botanical Garden, P.O. Box 299, St. Louis, MO 63166-09-299, USA.

${ }^{3}$ Autor para la correspondencia: thomas.croat@mobot.org.
} 
y las condiciones geográficas, la presencia de tres cordilleras hacen que la diversidad de ellas sea alta y en algunos casos estas se convierten en barreras ecológicas, aumentando de esta manera el endemismo de las especies. Los andes colombianos presentan aráceas desde el nivel del mar hasta alturas de los 3800 metros, este aspecto también limita la distribución de las especies incluso dentro de la misma cordillera, pero de manera sorprendente el Jardín Botánico San Jorge ha logrado establecer en un rango altitudinal de los 1200 metros una colección con 110 géneros de esta familia, que son de diferentes grados altitudinales. En Colombia se encuentran regiones de alta humedad, y regiones con bosques inalterados, convirtiendo a Colombia en la zona más rica en aráceas (Croat 1992). La diversidad de especies disminuye sustancialmente a mayor altitud incluso en bosques muy húmedos, pero es moderadamente rica a 1.100 metros como mínimo. La Cordillera Central de Colombia es sustancialmente más seca, particularmente durante parte del año y tiene más generalizadas, menos especies (Croat 1990).

\section{Descripcíon del area}

El departamento del Tolima se encuentra ubicado en centro de Colombia, localizado entre los $02^{\circ} 52^{\prime} 59^{\prime \prime}$ y $05^{\circ} 19^{\prime} 59^{\prime \prime} \mathrm{N} 1$, y los $74^{\circ} 24^{\prime} 18^{\prime \prime}$ y $76^{\circ} 06^{\prime} 23^{\prime \prime}$ W. Cuenta con una superficie de 23.582 $\mathrm{km}^{2}$ lo que representa el $2.1 \%$ del territorio nacional. En el departamento de Tolima se diferencian cuatro áreas climáticas: una semihúmeda localizada en la parte alta de la cordillera Central y Oriental, que tiene precipitaciones superiores a los 2.000 $\mathrm{mm}$ anuales. De oeste a suroeste se distingue una pequeña área catalogada como ligeramente húmeda, con un rango de precipitaciones de 1.500 a $2.000 \mathrm{~mm}$; esta misma unidad se extiende longitudinalmente sobre ambos piedemontes. Sobre el valle del río Magdalena se tipifica un sector subhúmedo, con precipitaciones entre 1.000 y $1.500 \mathrm{~mm}$ y temperaturas medias anuales superiores a $\operatorname{los} 24^{\circ} \mathrm{C}$.

Ibagué, capital del Departamento de Tolima, se encuentra localizado en las coordenadas geográficas: $4^{\circ} 15^{\prime}$ a $4^{\circ} 40^{\prime} \mathrm{N}$, y $75^{\circ} 00^{\prime}$ a $75^{\circ} 30 \mathrm{~W}$; tiene una extensión de $1.439 \mathrm{~km}^{2}$. Es atravesado en sus costados oriental, occidental y norte por los ríos Combeima, Chípalo y Alvarado, respectivamente. El río Combeima nace en las laderas del nevado del Tolima, y de los $5200 \mathrm{~m}$ de altura descienda a 1200 metros este descenso es lo que conocemos como el cañón del Combeima en la cordillera central, existen algunos poblados ubicados en este sector como Juntas, villa Restrepo, Chapetón, y la reserva natural Ibanasca muy importante en la conservación de la biodiversidad de la zona del Combeima, ella consta de 1800 hectáreas de bosque alto andino donde fueron encontrados dos de los nuevos anthurios estudiados.

\section{Métodos y Material}

Para la realización del siguiente estudio se procedió a la recolección de material vivo de campo del sector del cañón del Combeima, se llevaron los ejemplares vivos a la colección del jardín Botánico San Jorge, donde se realizó el seguimiento por un periodo de dos años, y también se entregaron muestras al herbario de TOLI y JAUM (herbario del Jardín Botánico de Medellín) y en la actualidad se tienen muestras vivas en la colección del jardín botánico San Jorge de la ciudad de Ibagué.

Anthurium combeimense Croat \& Oyuela, sp. nov. Tipo: COLOMBIA. TOLIMA: Municipio Ibagué, Sector Cañón del Río Combeima, Reserva Natural Ibanasca (El Palmar), km 4.6 de Ibague along road to Nevada, vic. de Villa Restrepo, $04^{\circ} 32.45^{\prime} \mathrm{N}$, $75^{\circ} 18.684^{\prime} \mathrm{W}, 1,600$ m. 15.XI.2008, fl., Croat \& Oyuela 100536 (holotipo, JAUM!; isotipo, TOLI!).

Figs. 1a-d, 3a

Anthurium combeimense differs from other species described from middle elevations in the foothills of the Eastern Andes. Anthurium paloraense Croat from $875 \mathrm{~m}$ in Morona-Santiago Province in the north central part of Ecuador is similar in having short, D-shaped petioles and in drying moderately dark but differs in having proportionately longer blades (5.5 times longer than broad versus 3.8 times longer than broad for A. paloraense) the primary lateral veins spreading at $35-40^{\circ}$ angle and drying blackened (versus at $60-75^{\circ}$ angle and brownish for $A$. paloraense) as well as by having a pale creamy yellow spadix.

Habito epífito en arboles ("arracacho"Vochysia amegalantha Staff.) de 8-15 metros de altura, pero también terrestre en pendientes superiores a $45^{\circ}$, en bosque subandinos en la cordillera central de Colombia (1600 a 2000 m.s.n.m.); Raíces en forma masiva y expuestas, blanquecinas, formando una especie de nido de pájaro, tallos con entrenudos muy cortos; catafilo ca. 9,5 cm de largo, $4 \mathrm{~cm}$ de ancho en la base, verde claro, este dura largo tiempo y luego se deshace persistiendo débilmente 


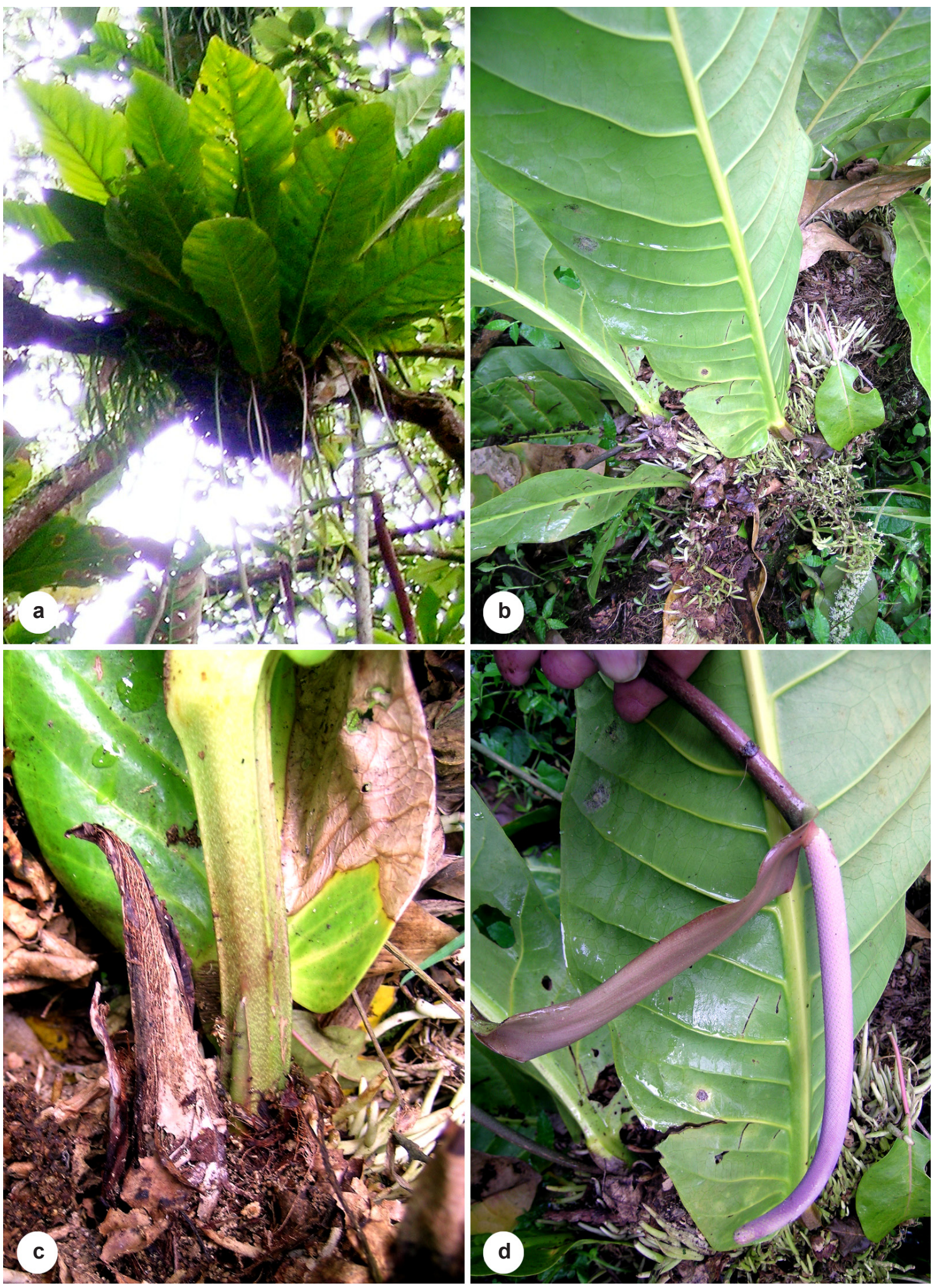

Figure 1 - New species of Anthurium (Araceae) from Colombia, Tolima, Ibagué, Cañon del Combeima. a-d. Anthurium combeimense Croat \& Oyeula - a. habit; b. leaf blade, abaxial lower surface; c. inflorescence, close-up; d. cataphylls and petiole. 
como fibras de color marrón. Hojas 9-12 por planta, 57-113 cm largo hasta $54 \mathrm{~cm}$ de ancho, acomodadas en roseta densa, saliendo hacia arriba y no pendiendo, pecíolos 9-16 cm de largo, 2,5-3 $\mathrm{cm}$ de diámetro, en forma de $\mathrm{D}$, aplanado aguda adaxialmente, con una costilla obtuso medial abaxialmente y las costillas 1 o 2 romo en la media inferior de cada lado, de color verde oscuro, con manchas de color café, secado grueso acanalado, café intermedio; geniculo ca. $7 \mathrm{~cm}$ de largo por $2.4 \mathrm{~cm}$ de diámetro, mostrando una coloración verdosa y teñido de café semejando cicatrices en la parte externa conformando un verdadero codo que le permite a la hoja quedar parada. Hojas lanceoladas a ovadas, acuminadas en el ápice de ca. $1 \mathrm{~mm}$ de apiculado, subcordadas en base con lados desiguales, 2.5-2.7 veces más largo que ancho, 4.85.2 veces más largo que el pecíolo, coriáceas, haz verde con un lustro muy suave, moderadamente, secado semi-brillante, de color amarillo oscuro y verde en la superficie superior, el envés verde claro o amarillo verdoso, sin lustre; visos ocres en la superficie de la hoja cuándo en el sol; nervio central aguda ovado anterior, más marcado en las dos partes centrales, en la primera y la cuarta parte muestra una forma redondeado y más delgado; venas laterales primarias 15 pares, derivadas de la nervadura central de $60-80^{\circ}$ y curvado hacia arriba, por poco redondeada y gruesa en ambas superficies, secado ligeramente redondeada, del mismo color en la superficie superior, de forma visible y agudamente planteado en la superficie inferior, el nervio colector producido por las venas laterales primarias, 2-4 mm desde el margen, el secado y discreta del mismo color. Inflorescencia, hasta $135 \mathrm{~cm}$ de largo, tendido y colgante, pedúnculo 56-78 $\mathrm{cm}$ de largo, 0.7-2 $\mathrm{cm}$ de diámetro, de forma redonda, de color verde oscuro, secado medio gris-marrón; espata de textura coriácea, hasta $54 \mathrm{~cm}$ de larga, $6 \mathrm{~cm}$ de ancha; extendiéndose débilmente, verde con tintes morados mostrando líneas a su largo, la inserción en el pedúnculo lo hace de manera decurrente; espádice hasta $54 \mathrm{~cm}$ de largo, $3 \mathrm{~cm}$ de diámetro, cónico, de coloración blancuzca al comienzo y verde claro después de antesis. Frutos rojo a purpura a medida que se maduran y caen de el espádice, ovados alargados, una o dos semillas blancas o cremas, envueltas por un sustancia gelatinosa y de sabor dulzón.

Anthurium combeimense es endémica de Colombia, conocida sólo de la localidad tipo en el departamento del Tolima en la Reserva Natural el
Ibanasca, a lo largo del cañón del río Combeima a $1600 \mathrm{~m}$ en una zona de vida de bosque húmedo pre-montano.

La especie es un miembro de la sección Pachyneurium caracteriza por su hábito epífito, entrenudos cortos, sistema de raíces masiva, catafilos lanceolados que persisten débilmente como fibras de color marrón; pecíolos en forma de $\mathrm{D}$ con una costilla medial obtuso y uno o dos costillas contundente en la mitad inferior de cada lado, denominados anthurios "nido de aves" para Colombia denominados "col de monte" y es un grupo natural. La mayoría tienen un corto hábito de crecimiento arocetado, hojas generalmente de gran tamaño que son más o menos obolanceoladas u obovadas (más ancho por encima de la mitad de la hoja), sushojas de secado amarillenta marrón claro.

Anthurium combeimense debe distinguirse de tres miembros de la sección Pachyneurium que también se producen en la misma región de Colombia: A. caucavallense Croat, A. glaucospadix Croat y A. paloraense Croat. Anthurium caucavallense presenta catafilos en forma de gancho, hojas enormes $100-200 \mathrm{~cm}$ de largo y espádices que van de 20-40 $\mathrm{cm}$ de largo, claramente cónica y que son de color verde azulado y aparecen glaucos. Anthurium glaucospadix Croat, difiere en tener hojas estrechas, ovaladas con las bases que son muy largas atenuadas en contraste con las bases de la hoja sub-cordadas de A. combeimense, también difieren en sus peciolos que son más largos y más delgados y también en el tamaño de la planta $A$. glaucospadix es más pequeña. Anthurium combeimense también debe ser comparada con otra especie descrita de elevaciones medias en las estribaciones de los Andes orientales: A. paloraense Croat, de $875 \mathrm{~m}$ en la provincia de Morona-Santiago, en la parte centro-norte de Ecuador. Es similar a A. combeimense en tener corto los pecíolos y en forma de $\mathrm{D}$ y hojas lanceoladas con venas extendiendo a $60^{\circ}-75^{\circ}$ de ángulo. Anthurium paloraense se distingue por las láminas de las hojas con bases atenuadas y estas se vuelven negras cuando se secan. La especie lleva el nombre de la localidad tipo a lo largo del Rio Combeima, en el departamento de Tolima.

Anthurium oyuelae Croat, sp. nov. Tipo: COLOMBIA. TOLIMA: Ibagué, Sector Cañón de Río Combeima, Reserva Natural Ibanasca (El Palmar), distancia del sitio de colecta al parque central de Ibagué, $31 \mathrm{~km}$ Ibagué, punto de colecta frente a Hotel Iguaima, $04^{\circ} 34^{\prime} 49^{\prime \prime} \mathrm{N}, 75^{\circ} 19^{\prime} 35^{\prime \prime} \mathrm{W}$, 
elev. 2,300 m, 15.XI.2008, fl., Croat \& Oyuela 100540 (holotipo, JAUM!; isotipos, COL!, TOLI!).

Fig. 2a-d

The species is best compared to Anthurium nymphaeifolium $\mathrm{K}$. Koch \& Sello which ranges from Venezuela through the Cordillera Oriental and reaches the Cordillera Central. That species differs in having the epidermis drying moderately smooth or minutely granular and a spadix that is cylindroid or cylindroid-tapered and typically white or cream at anthesis. In contrast $A$. oyuelae has blades that dry markedly areolate-wrinkled on upper surface with the pale linear cellular inclusions mostly sunken on the upper surface (in contrast to being essentially flat or weakly raised on the surface for $A$. nymphaeifolium).

Plantas epífitas, ubicado a alturas de $2-3 \mathrm{~m}$ del suelo, o terrestre en laderas con alta inclinación, poco expuestas al sol pero no en el interior del bosque; raíces gruesas, $0.5-0.7 \mathrm{~cm}$ de diámetro, color cafés o rojizas y a medida que el tallo se, tallos hasta 1 metro de largo verdes, siempre con rezagos del catafilo, ocres, hojas enteras retorcidas y no en fibras; entrenudos cortos, ca. $4 \mathrm{~cm}$ por 3-6 $\mathrm{cm}$ de diámetro; catafilos 15-22 cm de largo, lanceolados, verde pálido, secado medio gris-marrón o ocre, persistindo intacto, formando una estructura anexa fibrosa.

Hojas 12-14 por planta al final del tallo. Pecíolo ca. $85 \mathrm{~cm}$ de largo, 6-7 $\mathrm{mm}$ de diámetro cuando fresco, secado de ca. $5 \mathrm{~mm}$ de diámetro a mitad de este, cilíndricos, verde medio y semibrillante, el secado de manera restrictiva y obtusamente sulcados, medio gris-café y mate, ligeramente más delgado en la parte adaxial; genículo ca. $4.5 \mathrm{~cm}$ de largo, ligeramente más oscuro que el pecíolo; vaina roja, convoluta, hasta $12 \mathrm{~cm}$ de largo; láminas ovado-triangulao, de 36-46 cm de largo, 16.5-24.5 $\mathrm{cm}$ de ancho, 1.8-2.2 veces más larga que ancha, subcoriáceas, semibrillante verde oscuro por el haz, secado medio gris marrón pálido, y verde más claro y mate por el envés, secado medio por debajo de color amarillo-marrón, lámina con lóbulo anterior ca. $46 \mathrm{~cm}$ de largo, margen fuertemente convexa; lóbulos posteriores $10-12.5 \mathrm{~cm}$ de largo, ca. $12 \mathrm{~cm}$ de ancho, seno obovado, 9-10 $\mathrm{cm}$ de profundidad, ca. $3 \mathrm{~cm}$ de ancho; nervadura central estrechamente redondeada que a medida que se aleja de la base se torna más delgada, perdiéndose en el ápice; venas laterales primarias presentan tres pares, en un ángulo de 55-64 ${ }^{\circ}$, débiles hundidas y del mismo color del haz, redondeadas y delgadas por el envés y del mismo color de este; venas terciarias estrictamente convexas y más oscuras por el envés y por el haz un poco más claras; vena colectora son producidos por las venas mediales laterales primarias, $8-10 \mathrm{~mm}$ desde el margen; venas basales de 4 a 5 pares, los 2 primeros pares libres a la base, el tercer par fusionado a ca. $1.5 \mathrm{~cm}$, el 4 y 5 se unen a ca. $2.7 \mathrm{~cm}$, la costilla basal a ca. $2.7 \mathrm{~cm}$ de largo, desnudo hasta $1.3 \mathrm{~cm}$, claramente aguda, verde claro ocasionalmente con unos visos rojizos. Inflorescencias erectodivergentes; pedúnculo $45-85 \mathrm{~cm}$ de largo, 4-6 mm de diámetro a medio camino, más delgada cerca de el espádice, algo más corto que el pecíolo, de manera restrictiva y obtusamente verde sulcados, medio y mate cuando está fresco, secado medio y marrón más claro y mate, pedúnculo de 3.6 veces más largo que la espata; espata reflexa, 10.7-18.5 cm de largo, 3.6-4 cm de largo, el secado de 1.7-1.9 $\mathrm{cm}$ de ancho cerca de la base, oblongo y caudatoacuminado este de $1.4 \mathrm{~cm}$ de largo, presenta en el interior una textura subcoriácea, violeta-púrpura un poco brillante, la parte externa verde claro, con líneas muy delgadas purpuras y en general sin brillo o mate, secado medio marrón rojizo; espádice sésil, 17-21 $\mathrm{cm}$ de largo y 11-15 mm de diámetro en la base y medio y diámetro de ca. $7 \mathrm{~mm}, 1 \mathrm{~cm}$ del ápice en estado fresco, secado ca. $16.5 \mathrm{~cm}$ de largo y $9 \mathrm{~mm}$ en la base y $8 \mathrm{~mm}$ en el centro y $6 \mathrm{~mm}$ diámetro, 1 $\mathrm{cm}$ del ápice, cilíndrico, disminuyendo ligeramente en el ápice, el ápice redondeado, medio y oscuro mate de color púrpura, con pistilos débilmente que sobresalen, polen amarillo pálido, flores visible por espiral 8-9, 2.6-2.7 $\mathrm{mm}$ de largo, 2.2-2.5 $\mathrm{mm}$ de ancho, tépalos 1.8-2 $\mathrm{mm}$ de ancho, margen externo de 2 caras, el margen interior derecho; estambres a cabo en el nivel de los tépalos, ca. $0.6 \mathrm{~mm}$ de largo, ca. $0.7 \mathrm{~mm}$ de diámetro; tecas ligeramente bifurcado.

Anthurium oyuelae es endémica de Colombia, conocida sólo de la localidad tipo en el departamento del Tolima a $2300 \mathrm{~m}$ de un bosque montano bajo húmedo o zonas de vida bosque húmedo montano.

La especie es un miembro de la sección Calomystrium caracterizado por su hábito epífito, entrenudos cortos, persistentes catafilos intactos, laminas estrechamente ovado-sagitadas de color marrón-secado las hojas con un seno espatuladas, dos pares libres de venas basales, las venas colectivos producidos por una de las venas laterales primarias, así como por el largo pedunculadas inflorescencia con una espata verde reflejos púrpura y una espádice cónico débilmente. Especialmente característicos son las alineaciones de corto, claro que están hundidos en las cavidades de hendidura en la superficie superior. 

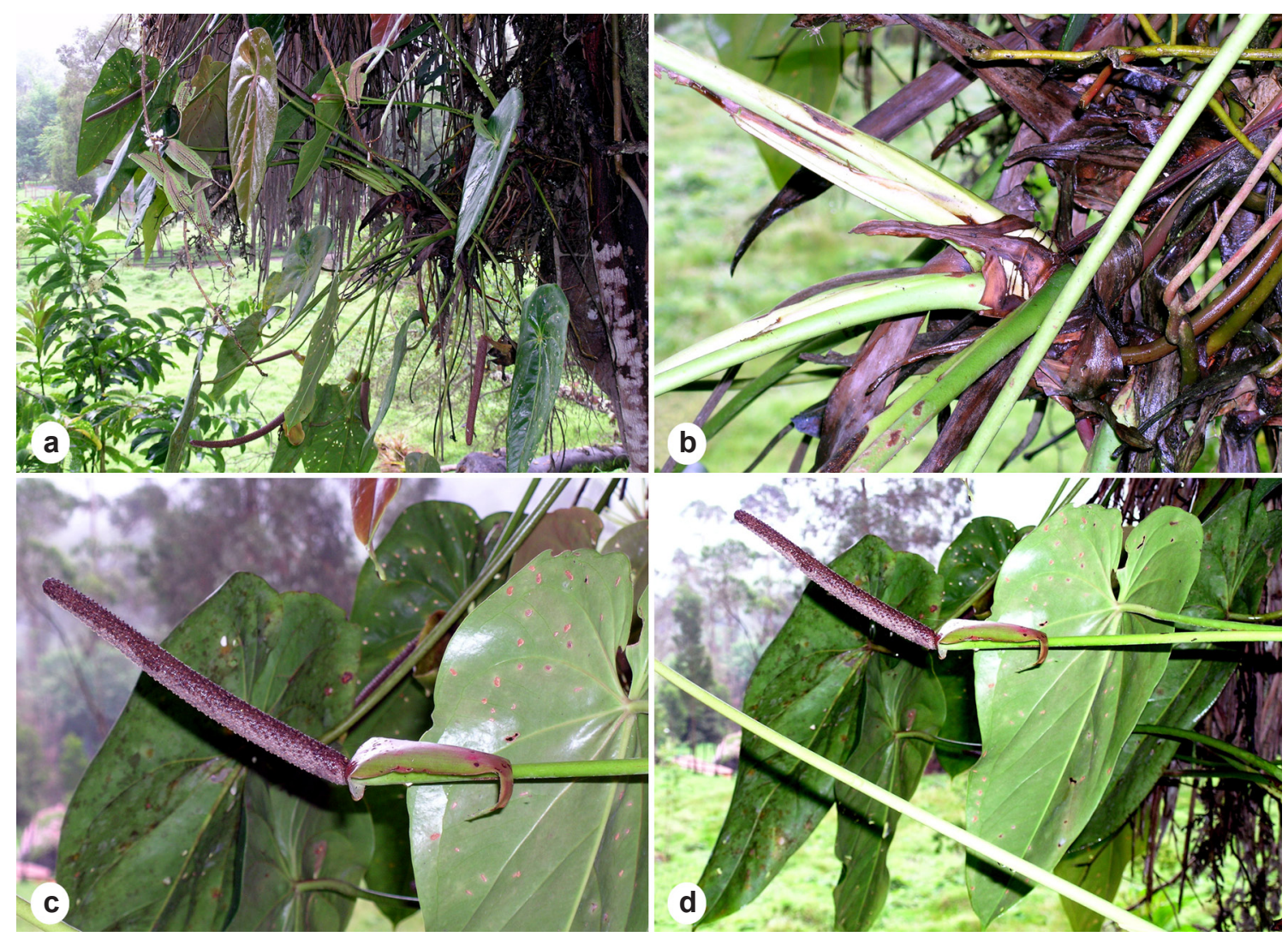

Figure 2 - New species of Anthurium (Araceae) from Colombia, Tolima, Ibagué, Cañon del Combeima. a-d. Anthurium oyuelae Croat - a. habit; b. stems and cataphylls, close-up; c. Inflorescence, close-up; d. leaf blade, adaxial surface with inflorescence.

La especie se puede confundir con Anthurium nymphaeifolium $\mathrm{K}$. Koch y Sello, que va desde Venezuela a través de la Cordillera Oriental y llega hasta la Cordillera Central. Esa especie se diferencia en tener la epidermis con un secado moderadamente suave o granular minuciosamente y un espádice que va de cilíndrica a cilíndrica afilado y por lo general de color blanco o crema en la floración. Por el contrario Anthurium oyuelae tiene hojas que se secan marcadamente areoladasarrugas en la superficie superior con la pálida inclusiones lineales celulares sobre todo hundido en la superficie superior (en contraste con ser esencialmente planas o ligeramente elevadas en la superficie de $A$. nymphaeifolium. La especie fue nombrada en honor al coautor de esta publicación, el botánico colombiano, German Orlando Oyuela Torres. German es Director del Jardín Botánico San Jorge. German es muy versado de la flora y fauna de la Reserva de Ibanasca Natural donde realizó estudios durante años. Desarrolló casi solo de por sí una de los más interesantes y diversas colecciones de plantas vivas vista en América del Sur. Su colección de Araceae es excelente y esta especie preciosa que lleva su nombre será una adición bienvenida.

Anthurium sanjorgense Oyuela \& Croat, sp. nov. Tipo: COLOMBIA. TOLIMA: Parque Nacional de los Nevados, zona de amortigison, vic. El Silencio, at end of road between Ibague and park, along trail to La Cabaña, ca. $0.2-5 \mathrm{~km} \mathrm{~N}$ of end of road, 04³5'59.4'N, 75'19'49.1'W, 2609 m, Croat, G. Ferry, D. Scherberich \& Oyuela 103882 (holotipo, JAUM;isotype:TOLI).

Fig. 3b-d Anthurium sanjorgense is similar to $A$. oyuelae in terms of size and texture and coloration of the leaves but the inflorescence differs in coloration of the spathe with $A$. sanjorgense having a bicolorous spathe with the lower half violet-purple and the apical half green. Anthurium sanjorgense is also confused with A. oxybelium 
Schott, differing from that species by the texture of the leaves and the position of the spathe as well as by the prominently exserted stamens in the latter. Anthurium variegatum differs by having a purple spathe with prominent green veins. In contrast to both $A$. oxybelium and $A$. variegatum, the spathe of A. sanjorgense is decidedly bicolorous.

Planta de corte hemiepifito, sub-rastrero, tallos alargados y rastreros que intentan sobresalir en busca de la luz, raíces de coloración grisácea, saliendo de los entrenudos, redondas, que alcanzan los $0.5 \mathrm{~cm}$ de diámetro, entrenudos cortos de 2-6 $\mathrm{cm}$ de largo, 2-3 $\mathrm{cm}$ de diámetro $\mathrm{y}$, totalmente verdes en plantas adultas, pero en plantas relativamente jóvenes presentan una coloración marrón o verdes con visos vino tintos; catafilos de 16-20 cm de largos, verde claro, al secado marrón, persistentes, envolviendo el tallo sin caerse y no se convierten en fibras. Hojas por lo general 4 a 6 , que se acomodan a lo largo del tallo de forma alternada; peciolos 78-84 cm de longitud, ca. 0.8 $\mathrm{cm}$ de diámetro, con poca variación en el grosor entre la parte basal y la apical, cilíndrico, verde claro cuando aún están jóvenes, coloración verde con visos marrón cuando maduros, sulcado y de coloración marrón mate cuando seco; geniculo marcado que alcanza los $3.5 \mathrm{~cm}$ de longitud, ligeramente más grueso que el peciolo, obtuso y redondo, verde claro, café-grisáceo y notoriamente sulcado cuando seco; lamina lanceoladas (ovada triangular), simples de bordes enteros, con lóbulos posteriores que alcanzan cada $8-9 \mathrm{~cm}$ de ancho por ca. $10 \mathrm{~cm}$ de largo, seno que alcanza los 10 $\mathrm{cm}$ de profundo, por $2.5 \mathrm{~cm}$ de ancho, verde mate sin lustre, un poco más clara en el envés y textura simi-coriácea hasta $33 \mathrm{~cm}$ de largo por $13 \mathrm{~cm}$ de ancho, 13-14 nervaduras secundarias por cada lado, nervio colector notorio a ca. $0.7 \mathrm{~cm}$ del borde de la hoja, que sale de la cuarta nervadura basal, muestra tres nervaduras basales muy marcadas, las nervaduras terciarias forman una red pero poco visibles en las hojas vivas mientras que en las hojas secas se notan más, base bilobulada de forma espatulada lóbulo alcanza los $10 \mathrm{~cm}$ ancho y un ápice acuminado. Inflorescencia presenta un pedúnculo de 70-74 $\mathrm{cm}$ de longitud, mostrando al final de la parte apical de este una formación a manera de cuello de ganso, verde claro con algunos visos vinotintos, no superando $0.8 \mathrm{~cm}$ de grosor, redondo, liso; la espata y el espádice se encuentran ubicados en forma decurrente espata vinotinta a rojiza en su parte basal, que alcanza a llegar hasta la mitad de esta y el resto es de coloración verde claro, la espata hasta $1 \mathrm{~cm}$ largo por $4 \mathrm{~cm}$ de ancho, lanceolada; espádice cilíndrico, pedúnculo ca. $3 \mathrm{~cm}$ de largo, purpura o vino tinto, espádice hasta $7 \times 1 \mathrm{~cm}$, cilindroide, stipitata a $3.2-4 \mathrm{~cm}$, el porción con flores $6-7 \mathrm{~cm}$ largo, ca. $1 \mathrm{~cm}$ diámetro, purpureo a purpureo-violeta; flores ca. 4 per espiral, ca. $1.4 \mathrm{~mm}$ largo y ancho; tepalas ca. $0.7 \mathrm{~mm}$ de ancho, margen externo de 2 caras, el margen interior rodundiado; estambres a cabo en el nivel de tepalas, ca. $0.6 \mathrm{~mm}$ largo, ca. 0.7 $\mathrm{mm}$ ancho; tecas ellipsoide, ligermente bifurcado.

Anthurium sanjorgense es endémica de Colombia, conocida sólo de la localidad tipo en el departamento del Tolima en la Reserva Natural el Ibanasca, a lo largo del cañón del río Combeima a 2600 metros en una zona de vida de bosque húmedo pre-montano en el sector del cañón del rio Combeima denominado como el rancho vía al nevado del Tolima en un bosque alto andino, o bosque de niebla. El lugar de su ubicación es muy extremo por la baja intensidad lumínica del sector y la alta humedad en el ambiente, este lugar está muy cerca de la zona demarcada como zona de amortiguación del parque los nevados del sector del nevado del Tolima. La especie lleva el nombre sanjorgense por que ha sido dedicado al jardín botánico San Jorge de la ciudad de Ibagué, fue la entidad encargada de hacer los estudios sobre la zona donde se encontró y es el único jardín que lo tiene en la actualidad en colección.

Anthurium sanjorgense difiere de Anthurium oyuelae en el tamaño, textura y coloración de las hojas, la inflorescencia difiere en la coloración de la espata ya que en $A$. sanjorgense presenta dos colores y $A$. oyuelae solo un color, pero podría confundirse con $A$. oxybelium, de este se diferencia por la textura de las hojas y definitivamente la posición de la espata y su coloración lo hacen fácil de distinguirlo, con $A$. variegatum podría tener cierto parecido pero $A$. variegatum presenta una coloración muy purpura con lineas verdes en su espata mientras que $A$. sanjorgense presenta la espata con dos colores.

Anthurium sanjorgense pertenece a la sección la Calomystrium, presenta las siguientes características las hojas son relativamente coriáceas y secan marrón. Pertenece al grupo de los anthurios 

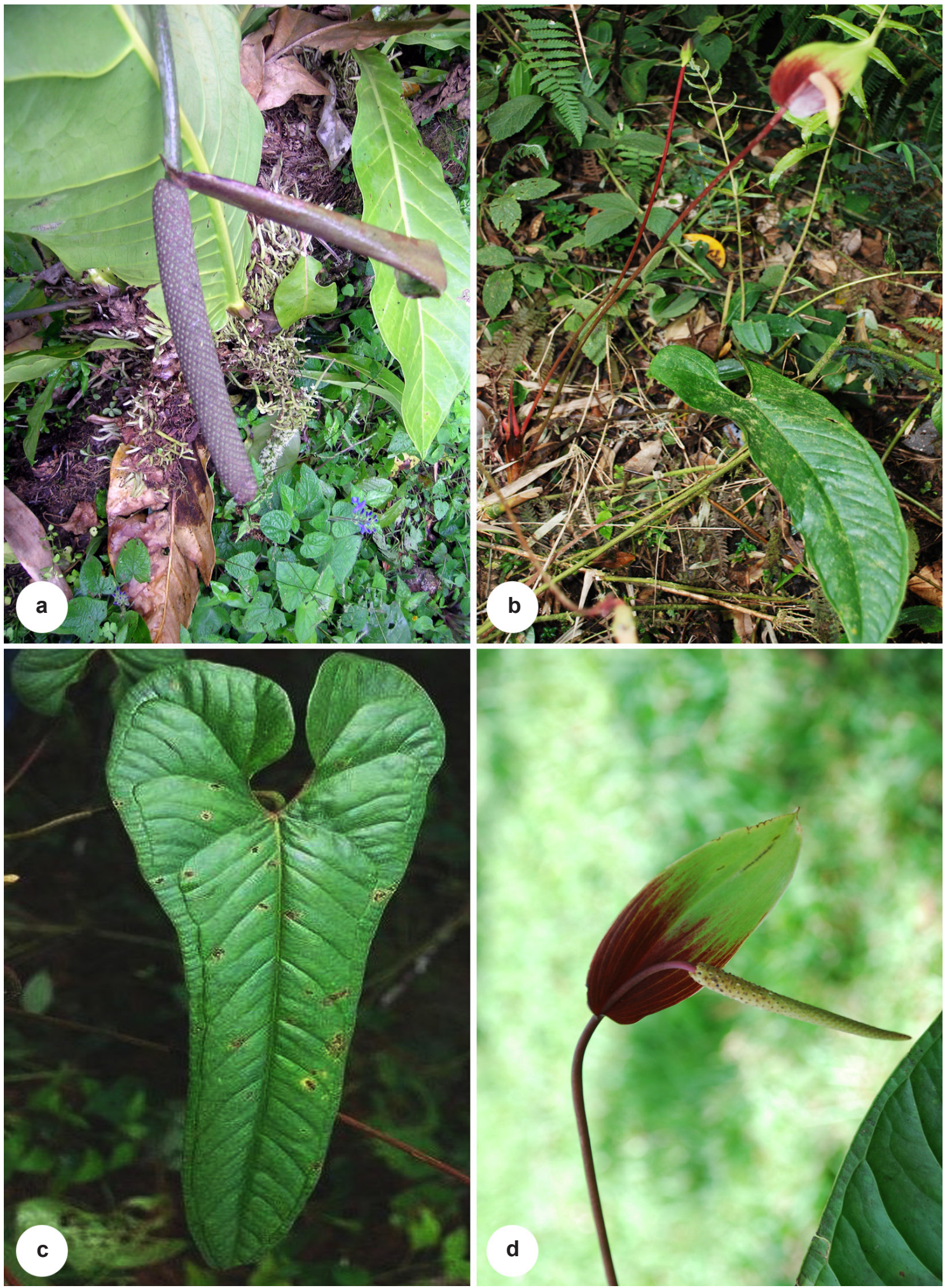

Figure 3 - New species of Anthurium (Araceae) from Colombia, Tolima, Ibagué, Cañon del Combeima. a. Anthurium combeimense Croat \& Oyuela - immature infructescence. b-d. Anthurium sanjorgense Oyuela \& Croat - b. habit; c. leaf blade, adaxial surface; $d$. inflorescence. 
que se producen en altitudes relativamente altas y tienen las espatas greusas y colorada, y catafilos persisten intacta (Croat \& Sheffer 1983). Estas especies se ajustan a la circunscripción original de Schott de la sección.

\section{Referencias}

Boyce, P.C. \& Croat, T.B. 2011 onwards.The Überlist of Araceae, totals for published and estimated number of species in Aroid genera. Available in $<$ http://www.aroid.org/genera/140601uberlist. pdf $>$. Access on 5 February 2012.
Croat, T.B. 1988 (1990). The ecology and life forms of Araceae. Aroideana 11: 4-56.

Croat, T.B. 1992. Species diversity of Araceae in Colombia: a preliminary survey. Annals of the Missouri Botanical Garden 79: 17-28.

Croat, T.B. \& Sheffer, R.S. 1983. The sectional groupings of Anthurium (Araceae). Aroideana 6: 85-123.

Mayo, S.J.; Bogner, J. \& Boyce, P.C. 1997. The genera of Araceae. Royal Botanic Gardens, Kew. 370p.

Temponi, L.G. \& Coelho, M.A.N. 2011. Two new species of Anthurium sect. Urospadix (Araceae) for Brazil. Rodriguésia 62: 315-320. 
